
record.

\title{
Accessing Populations with Specialized Clinical Needs: An Illustrative Case Study using Google Adwords ${ }^{\mathrm{TM}}$
}

\author{
Warren O. Eaton and Katherine M. Kenyon \\ University of Manitoba
}

\begin{abstract}
Author Note
Warren O. Eaton, Department of Psychology, University of Manitoba; Katherine M. Kenyon, Department of Psychology, University of Manitoba.

This work was supported by an award from Google Grants Adwords for Non-Profits program to the Milestones Centre, University of Manitoba. We wish to thank Edward Johnson, Colleen Singbeil, and Diane Hiebert-Murphy for their comments on an earlier draft of this paper.

Correspondence concerning this article should be addressed to Warren O. Eaton, Department of Psychology, University of Manitoba, Winnipeg, Manitoba, Canada, R3T 2N2. E-mail: warren.eaton@umanitoba.ca
\end{abstract}

\begin{abstract}
When seeking help for health problems, a majority of individuals now look online first. There they are confronted with millions of results and typically visit only a few top-listed websites. As a consequence, being noticed is a significant problem for psychologists who use online tools to recruit help-seeking persons to either research studies or professional practices. The salience of a psychologist's online presence can be increased through the use pay-per-click (PPC) advertisements that, for a price, appear near the top of the search results listings. The potential of PPC advertising to recruit a clinical sample for a research survey on childhood obsessive-compulsive disorders (OCD) was tested in this proof-of-concept case study using Google Adwords ${ }^{\mathrm{TM}}$. We created and implemented ads whose appearance on searchers screens was triggered by selected keywords that matched the content of our website landing page. Ads were displayed in 11 selected geographic locations worldwide and were aimed at parents of children with OCD. Over a 16-week campaign 183 participants completed our survey at an average cost of $\$ 27$ per survey completion. Detailed keyword phrases were most effective, and geographical variation was notable. The results show that PPC advertising can be cost effective for the recruitment of participants to studies on a specialized clinical topic. Moreover, because PPC ads can be restricted to small geographic areas, online advertising may also be cost effective for making psychological services known to potential local clients.
\end{abstract}

Keywords: telepractice, advertising, obsessive-compulsive disorder, children, pay-per-click, PPC, click-through, conversion, geo-location

Telepractice is possible because advances in technology have decreased the cost and increased the convenience of interactions at a distance. Psychologists, who have traditionally served local clients in face-to-face conversations, can now have 
Professional Psychology: Research and Practice

(C) 2013 American Psychological Association DOI: 10.1037/a0034505

This article may not exactly replicate the final version published in the APA journal. It is not the copy of record.

such interactions over great physical distance for little or no cost. Consequently, the historical constraints of clinician-client proximity can been broken, and telepractice has become feasible. Unlike the traditional situation in which clients learn of psychological services and information through word of mouth or local advertising, telepractice requires different mechanisms for client-professional connection. One such mechanism is provided by online search engines like Google, Bing, and Yahoo, which allow searchers in disparate locations to find highly specialized information and products, which are often referred to as the Long Tail (see Anderson, 2006). This potential connecting of searchers and providers in specialized niches sidesteps the need for expensive mass advertising that would have been required in the past.

Professional psychologists are specialized information providers, so if they develop a web presence, they could could use search engines to be found by potential research participants or clients who are searching the web for help with psychological problems.

Being found is more difficult than it first appears because many, if not most, online searches result in a tsunami of "hits." For example, a recent search on Google for "I need help for depression" produced 1.6 million results. A psychologist might have a wonderful web page about depression and its treatment, but the likelihood of it appearing on the first page of a online search for "depression" is essentially zero. This is bad news because searchers rarely go past the first 3-5 items on the first page of search results. More focused searches will reduce the number of results but, even so, it is extremely difficult to create website content that will lead to an appearance at the top of the search results list. These search results appear in the wide, leftmost column of a search engine's results page and are referred to as organic results. They are the product of the application of the search engine's algorithm to the body of information on the web, and no one has paid for their appearance.

In contrast, search results that look similar to organic results but appear in the rightmost, narrower column of Google search pages are advertisements. These ads appear because an advertiser has arranged with Google for their display whenever designated keywords or phrases are entered as search terms. A search-triggered ad, which appears on the searcher's screen along with competing ads and organic results, is called an impression. Typically, the advertiser doesn't pay Google for the impression ${ }^{1}$, but only pays if the searcher takes the action of clicking on the ad, hence the name pay-per-click (PPC) advertising.

When a searcher clicks on a PPC ad they are taken to the associated web page, and at that moment three parties benefit: Google gets paid by the advertiser; the advertiser has the searcher's attention; and the searcher may find some needed information. Because there are many fewer PPC search results than organic search results, it is far easier, though more expensive, for the information provider to be found through a PPC ad than through an organic search result. In effect, the PPC advertiser pays for a higher position and greater visibility on the list of search outcomes. That is immensely important because a prominent location on the search results page gets one noticed, whereas a poor location leaves one undiscovered. This PPC model of advertising is the revenue foundation of Google Inc's business, and it underwrites their many other free services such as Gmail (Google Inc., 2013).

\footnotetext{
${ }^{1}$ There are alternative forms of online advertising in which the advertiser pays a set amount for every 1000 impressions.
} 
This article may not exactly replicate the final version published in the APA journal. It is not the copy of record.

Online advertising may be wonderful for Google's business, but why is online advertising relevant to the professional psychologist? It is because individuals are strongly motivated to find solutions for their health problems, and $77 \%$ of them begin their search at a search engine (Fox \& Duggan, 2013), usually Google (comScore, 2013). Using the web in this way has a number of advantages for the user: it is anonymous, private, and free. Of course, what the searcher finds varies dramatically in quality. High quality, relevant information about mental health concerns can be provided by professional psychologists, but such high quality information is only useful if it can be found by the searcher. We argue here that psychologists may want to consider pay-per-click advertising to bring their information and knowledge to the attention of individuals who need their information and services.

We conducted an online research survey about a mental health problem, and what follows is a case study of how we recruited participants from around the world. The technological innovation that enabled us to recruit globally rather than locally is also very relevant to recruiting from a very specific target population, which we needed to do. PPC advertising appeared to have the potential for connecting our research study with potential participants who were searching online for information about the very topic of our research. The key point is that search engines can mediate the forging of a connection between us psychologists and individuals seeking information that we can provide. Pay-per-click advertising within the Google Adwords $^{\mathrm{TM}}$ (Google Inc, Mountain View, CA) framework offered a specific tool that we could use to that end.

One of us had had experience in the use of PPC for child development questions and had been using Adwords for a number of years. This experience provided us with insight about ways to reach a niche audience. We also had Adwords funding ${ }^{2}$ through a grant from Google Inc. that supports the use of advertising by non-profit organizations. Thus, we had the means to see if it is possible to reach a clinical sample using pay-per-click advertising, something we had never tried. The availability of funding for Adwords use was fortuitous because online searches are done using Google in $67 \%$ of cases (comScore, 2013). We had some experience and the necessary resources. Could we recruit an online clinical sample using PPC methods for a reasonable amount of money? The answer was far from clear at the outset because the online marketing world is a Darwinian one, where success is far from assured.

The aims of our study were to examine the critical issues of age at onset, symptom severity, and gender differences in obsessive-compulsive disorder (OCD) in children. First, it was hypothesized that age at onset would be negatively correlated with symptom severity. It was further hypothesized that males would experience an earlier age at onset than females, and thus more severe symptoms. Finally, it was also hypothesized that the proportion of male juveniles with OCD would exceed the proportion of female juveniles with OCD. The study addressed all three questions using an online survey. We wanted to obtain a larger, more heterogeneous sample than those employed in previous research with clinic samples. The

\footnotetext{
2 The grant funding consisted of Adwords credit, so we could only use Google PPC advertising; similar programs can be found with other search engine providers such as Bing and Yahoo. We have no financial interest in Google, Inc., and Google had no knowledge of, or influence over, the direction and content of our research.
} 
Professional Psychology: Research and Practice

(C) 2013 American Psychological Association DOI: 10.1037/a0034505

This article may not exactly replicate the final version published in the APA journal. It is not the copy of record.

target population was fairly specific, as we sought to recruit the parents and caregivers of children and adolescents under 16 years of age with OCD. We hoped that the online nature of the study would allow for the recruitment of a large, diverse sample of juveniles with OCD by collecting information from English-speaking individuals in a variety of countries around the world.

Our goal, then, was to conduct a proof-of-concept case study of the potential of Google adwords as the recruitment vehicle for a research survey on a specialized clinical question. We did not know if it would work at all, and if it did work, we did not know if it would be cost effective. In this regard, we had no a priori dollar-per-recruit threshold as being effective, but we were acutely aware of the financial constraints that most researchers face. We will describe our use of adwords to recruit participants to our study and the results we obtained. First, however, it is helpful to understand the Adwords system works, and we offer the following guide.

\section{Google Adwords Primer}

Milliseconds after one initiates an online search by entering a keyword or phrase in a web browser search box, a list of results appears in a series of entries on a series of pages. With few exceptions the ads that get noticed appear at, or near, the first listing on the first page. Whether a particular ad appears as an impression in those coveted top few spots is determined by an intersection of the behavior of the searcher, the choices of the advertiser, and the secret, proprietary search algorithms of the search engine. The needs of searcher and the advertiser are usually obvious. Less obvious to most is that Google's very survival depends its ability to please the searcher by taking them to good answers to their search questions (Collins, 2012). If searchers are repeatedly disappointed by being taken to irrelevant or poor sites, they'll abandon Google's search engine tool, and their multi-billion-dollar business would collapse. Because search result relevance is so important, we'll emphasize how it applies to the chain of steps between keyword entry and arriving at a particular landing page on the web.

Landing pages. These are the web destinations users reach when they click on PPC ads. Not every landing page is acceptable for Google ads. Some products and services are regulated by law, which can vary tremendously from country to country, and Google requires their ads to comply with laws and regulations. Indeed, Google's general rules for landing pages are often more restrictive than those demanded by particular laws because Google wants their users to have safe, legal experiences. The content of landing pages must also be consistent with the content of the triggering keywords and the clickable ad (e.g., a landing page about tires would not be acceptable as the destination for an ad about photography). As noted earlier, Google does not want users to have unexpected surprises when they click on an ad, and a prospective advertiser needs to align the content of the landing page with the content of their ad, and their keywords. Advertisers whose landing pages are highly relevant to their advertising content are rewarded by Google.

Keywords. Words and phrases used to initiate a search are called keywords, and they are the first link in the search chain that concludes at a landing page. Falling between keywords and landing pages are the actual Google ads. An ad's appearance on the user's screen occurs 
Professional Psychology: Research and Practice

(C) 2013 American Psychological Association DOI: 10.1037/a0034505

This article may not exactly replicate the final version published in the APA journal. It is not the copy of record.

because an advertiser has told Google to display it in response to triggering a keyword specified by the advertiser. Such an instruction would be based on the advertiser's belief that use of the keyword would imply searcher interest in content of the ad. That assumption may not always be correct, so a crucial task for the advertiser is to anticipate search terms a user might use.

Keyword tools. Free help is at hand with Google's Keyword Planner tool. With this tool, one can enter a potential search term like psychologist, and the Planner will provide lots of useful data on how frequently that term is actually used, synonyms, and related terms. This information can be quite detailed. For example, usage rates for a given search term or phrase can be obtained for a specific city or country. Synonyms and related terms and categories are suggested by Google to assist the advertiser in identifying a set of ad-triggering keywords. It behooves advertisers to remember, however, that Google is paid for clicks. It is in their interest, then, to suggest a large and broad set of keywords because the use of more keywords will result in more clicks and more revenue for Google. In fact, Google may suggest dozens or even hundreds of keywords as possible triggers for the ad's display.

Ads. Google ads are brief, substantially shorter than a Twitter tweet, and comprise a header line of 25 or fewer characters, two content lines of 35 or fewer characters each, and the landing page web address (URL). Moreover, each ad must meet strict requirements as to form (e.g., no exclamation marks in the heading) and content. Most crucially, ad content must be relevant both to the keyword that triggers it and to the landing page to which it leads. That required relevance means that what the user expects to find will have a meaningful connection with what they actually find. Expectations implied by the user's choice of search term must be consistent with the content of the ad and, if the ad is clicked, with the content of the landing page to which they are taken. Once an ad has been written and linked to keywords and landing page, it is submitted to Google for evaluation, a process that can take a few hours or some days. Google applies a set of requirements, some of which are clearly specified, some of which are proprietary and unknown, to an ad and renders a judgment. Once an ad has been approved, the advertiser can release it to the internet after making a variety of decisions about the conditions that apply to the ad's actual appearance on searcher's screens. A functioning online ad is only a means to an end, and in the online world that end is called a conversion.

Conversions. The point of advertising is to persuade someone to take some action, such as buying a product or making a donation. In our case, that action was completing our survey, which was operationalized as reaching the survey's final page. Adwords technology allowed us to generate a snippet of code, which we then placed on that specific webpage. Whenever a visitor reached that page the code signalled a conversion which could be linked both to the keyword that triggered an ad and to the ad that triggered the click. We could then see which of our keywords and ads were most effective in getting someone to complete our survey.

Bidding. There are no set prices for a single paid click in the Adwords sphere. Rather, Google has developed an auction system in which advertisers bid against each other for the ranking position an ad will fill in response to a particular keyword (recall that a highly ranked position in the list is crucial for success). Ads that appear in response to competitive search keywords will cost more than ads connected with less competitive keywords. Some of the most expensive keywords, like insurance, attorney, and loans cost more than $\$ 40$ per click 
Professional Psychology: Research and Practice

(C) 2013 American Psychological Association DOI: 10.1037/a0034505

This article may not exactly replicate the final version published in the APA journal. It is not the copy of record.

(Hitstudios, 2013). On the other hand, ads for less competitive terms can can cost less than $\$ 1$ per click.

Cost controls. The cost of a click for a particular ad is also based on Google's Quality (Q) score for the triggering keyword. Quality scores range from 1 to 10 , fluctuate, and are based on many variables (Check and Understand, 2013), including: account history, geographic location, past click-through rate, landing page quality, keyword/search relevance, and keyword/ad relevance. In general, higher quality scores reduce the cost of a triggered ad and improve an ad's position relative to competitor ads. Competition and quality fluctuate continually, so ad pricing is not static. In the face of such uncertainty, the advertiser specifies a maximum bid price that they are willing to pay for a particular keyword. Whether or not the price paid is that maximum or a lower price will depend on competitors' bids on the same keyword. In addition to specifying maximum bids for all keywords, the advertiser sets a total budget and a daily spending limit. Once the daily spending limit is reached, Google stops displaying an ad until the next day. Adwords campaigns provide the advertiser with a number of choices for how ads are presented as we illustrate below.

Campaign settings. An advertiser has considerable control over the conditions for ad presentation. The language of a user's search query or profile can be specified, as can the geographic location of the user. Location targeting can include entire countries or be specified more narrowly, down to a 3-mile radius around a designated address. Location is relevant to another setting, that for day and time. Scheduling can be narrowed to specific days and time intervals, although the default is to display ads throughout the day and week. Ads can also be limited to the type of device being used for the search (computers, laptops, mobile phones). Through the use of settings selections, the advertiser can narrow the conditions for triggering the display of an ad considerably.

\section{Study questions}

We knew that an Adwords campaign could be developed around keywords likely to be used by parents concerned with childhood OCD. What we did not know was whether it is practical to use an Adwords advertising campaign to recruit a clinical sample. Would such a campaign recruit enough participants for an adequate sample? What would the cost of such a campaign be? Could we successfully recruit participants from multiple countries?

\section{Method}

\section{Materials}

Advertisements. We created a number of advertisements designed to be triggered by keywords related to children's obsessive-compulsive disorder, such as "signs of OCD in children." We began with different ads, each highlighting a different aim or aspect of the study and each using different terms. Over the course of the first few weeks of the study, these ads were adjusted. The ones that resulted in the most click-throughs were kept while less 
Professional Psychology: Research and Practice

(C) 2013 American Psychological Association DOI: 10.1037/a0034505

This article may not exactly replicate the final version published in the APA journal. It is not the copy of record.

successful ads were taken offline. Between one and five ads were employed for the remainder of the study. The most popular ad used in this study read:

The Symptoms of Child OCD:

University Study About Child OCD-

For Parents of OCD Kids Under 16.

The survey. We created an online survey using the survey website SurveyGizmo (SurveyGizmo, Inc., Boulder, CO). The survey consisted of between 10 and 12 pages, with a maximum of 36 questions. Some pages and questions were skipped, depending on the participant's previous responses. The first questions pertained to the current age and sex of the child in question.

Another item asked if the participant's child experienced obsessions. If the participant indicated that their child does experience obsessions, they would then respond to five 5-point Likert-type questions about the severity of their child's obsessional symptoms. The participant also indicated whether their child experiences compulsions. Again, if the participant confirmed that their child experiences compulsions, they would respond to five 5-point Likert-type questions designed to assess the severity of their child's compulsive behaviours. Combined, these questions yielded a symptom severity score ranging from 0 to 40 .

Other survey items pertained to treatment of the child's OCD. For example: "Is (child's name) currently taking any medication to treat OCD?" Overall family functioning was another of our areas of interest as this was expected to correlate with the OCD severity ratings obtained. This was assessed by ascertaining participants' agreement with three short statements. Next, general knowledge about the participant was obtained in the survey section entitled "About You." In this section the researchers gained information about the participant's relationship to the OCD child and how much time he or she spends per week in the same household as the OCD child. In addition we asked about the participant's educational background, socioeconomic situation, and religion, as well as some basic information about some of his or her personality traits. The participant also answered the crucial questions about the child's age at OCD onset.

\section{Procedure}

When potential participants clicked on an advertisement about the study, they were directed to the Childhood OCD homepage, or landing page. The landing page provided the potential participant with more information about the study and about the researchers involved. There was also a contact form by which individuals were able to contact the researchers to share their questions or comments. The study's landing page contained information about OCD in general, the purpose of the study, and the participation criteria, all of which were described using as many of the keywords specified in Adwords as possible. This told Google that the information contained on the landing page and elsewhere was indeed pertinent to the Google searcher's query, and ensured that the keywords employed by the researchers received good quality scores. Quality scores are assigned by Google and can indicate how well a keyword matches the content of the landing page. High quality keyword scores are beneficial in a number 
Professional Psychology: Research and Practice

(C) 2013 American Psychological Association DOI: 10.1037/a0034505

This article may not exactly replicate the final version published in the APA journal. It is not the copy of record.

of ways. In particular, their use leads to the triggered advertisements being shown for less money and at in a higher position on the list of search results. In other words, keyword relevance is rewarded by greater ad visibility at a lower cost. Effective use of PPC advertising demands that the content promise of an ad is fulfilled by the content of the website to which the visitor is taken.

Parents who wished to participate in the study were next directed to the study's online informed consent page. Clicking on the OK button located at the bottom of the informed consent page indicated that the individual had understood the information about participation in the research project and agreed to participate in the study. The participant was then directed to the online survey. Following completion of the online survey the participant was taken to a Thank You webpage. This page had information about contacting a local health care professional if the parent had experienced any distress while completing the survey. It also explained again how to get in contact with the principal investigator should they have any questions or concerns.

Participants also were given the opportunity to provide their email addresses if they wanted to receive a summary of the findings following the conclusion of the study. If participants wished to provide their email addresses, they were directed to a secure website (http://www.mailchimp.com) that requires a double opt-in procedure to leave their email address. Moreover, their email addresses were not linked to their data.

\section{Ad Campaign Results}

\section{Participation}

Click-through. A crucial question was whether or not an adequate number of parents of children with OCD would participate. There are several parts to an answer. The first is the question of whether enough people click on our ads at all. During a 16-week period, our ads appeared 495,692 times (impressions) and were clicked 6,281 times for a click-through rate (CTR) of $1.3 \%$. This response to our ad meant that approximately 50 individuals visited our landing page daily. Whether or not such a CTR would be adequate depends on the percentage of visitors who take and complete the survey. It is to that retention issue we now turn.

Visitor progress. Between having our ad appear on a searcher's screen and a completed survey are many decision points for the visitor. At each step in the sequence, the visitor had to decide whether to leave or to continue. What happened at each transition in the sequence between the ad's appearance and the completion of the survey? To answer these questions, we combined information from Adwords, Google Analytics (a website analysis tool), and SurveyGizmo, the software service that implemented our survey. Table 1 displays each event in the sequence, the percentage of visitors who continued from that event, and where they went next. The overall pattern is one of a funnel, in which a large number of searchers narrows to a small number who continue to the last page of the survey.

\section{Table 1}

\section{Participant Retention from Ad Appearance to Survey Completion}


Professional Psychology: Research and Practice

(C) 2013 American Psychological Association DOI: 10.1037/a0034505

This article may not exactly replicate the final version published in the APA journal. It is not the copy of record.

\begin{tabular}{|c|c|c|}
\hline Event & $\%^{\text {a }}$ Continuing to the & Next Step \\
\hline Ad appears on screen & 1 & Click on ad \\
\hline Landing page appears & 18 & Click button to learn more \\
\hline Arrive at Consent Page & 45 & Click / consent button \\
\hline Arrive at first survey page & 54 & Answer 1 or more items \\
\hline Visit all survey pages & 60 & Reach Thank You Page \\
\hline
\end{tabular}

${ }^{a}$ Rounded to nearest $1 \%$.

Conversions. Of the 6,281 searchers who clicked on one of our ads, 183 continued through the funnel to the Thank You page, for a $2.9 \%$ conversion rate. Of course, not all of those who reached the last page provided complete data, but our experience suggests that half or more of those who reach the end of a survey will provide relatively complete data. Such conversion results are encouraging: a yield of 90-100 participants from 4 months of data collection. We also learned a great deal about the process, so these preliminary results will lead to refinements that should improve both our click-through and conversion rates.

\section{Cost}

Our average cost per click was $\$ 0.80$, which seems inexpensive. However, when many clicks are involved, things add up. In total we spent $\$ 5004$ to obtain 183 surveys, making the average cost per completed survey $\$ 27.34$. This cost is influenced by many factors, such as ad position, ad relevance, and competition. Our average ad position was 2.7 , which means it typically appeared in the second or third position in the list of ads. Had we bid more for our maximum bid price, we could have achieved a higher rank position, but at a higher cost. The $\$ 27$ amount is probably an underestimate of our true participant cost because not all visitors who reach the last page of the survey have answered all of the questions. The cost per useful data point will be unknown until the completion of the study, but $\$ 27$ would represent the low bound on that amount.

\section{Which ads worked best?}

Although we used 19 different ads, most were slight variants of others, and we typically showed from one to five ads at any one time. After showing an initial set of ads in a rotation, we switched to a display option that allowed Google to favor the best performing ad. We also monitored ad performance on a regular basis and discontinued those with CTRs of less the 1\%. Eventually, the most clicked ad, which was listed in the method section, was shown $99 \%$ of the time. This favored ad's CTR was $1.31 \%$ and the cost-per-click (CPC) for this specific ad was $\$ 0.86$. 
Professional Psychology: Research and Practice

(C) 2013 American Psychological Association DOI: 10.1037/a0034505

This article may not exactly replicate the final version published in the APA journal. It is not the copy of record.

\section{Which keywords worked best?}

We began by specifying 101 keywords and closely monitored the CTR for each keyword. We discontinued use of any keywords having CTR's of less than $0.40 \%$, which reduced our set of active keywords to 39 . The 10 best performing keywords are presented in Table 2 . The criteria for their selection was the number of conversions produced per thousand impressions. 
Professional Psychology: Research and Practice

(C) 2013 American Psychological Association DOI: 10.1037/a0034505

This article may not exactly replicate the final version published in the APA journal. It is not the copy of record.

Table 2

Ten Highest Converting Keyword Phrases per 1000 Impressions

\begin{tabular}{|l|c|c|l|l|c|}
\hline Keyword & $\begin{array}{l}\text { Conversions/ } \\
\text { 1K } \\
\text { impressions } \\
\text { (Cnv1K) }\end{array}$ & $\begin{array}{c}\text { Quality } \\
\text { Score (Q) }\end{array}$ & $\begin{array}{l}\text { CTR } \\
(\%)\end{array}$ & $\begin{array}{l}\text { Q x } \\
\text { CTR }\end{array}$ & $\begin{array}{c}\text { Cost per } \\
\text { Conversion } \\
(\$)\end{array}$ \\
\hline symptoms of ocd in children & 1.9 & 4 & 4.9 & 19 & 20 \\
\hline ocd symptoms in children & 1.8 & 5 & 4.4 & 22 & 17 \\
\hline OCD in Children & 1.6 & 7 & 5.6 & 39 & 23 \\
\hline ocd in kids & 1.2 & 7 & 3.0 & 21 & 17 \\
\hline Children with OCD & 0.9 & 4 & 3.6 & 14 & 29 \\
\hline ocd children & 0.6 & 4 & 1.3 & 5 & 18 \\
\hline OCD Symptoms & 0.5 & 4 & 1.5 & 6 & 27 \\
\hline $\begin{array}{l}\text { obsessive-compulsive } \\
\text { disorder in children }\end{array}$ & 0.4 & 7 & 1.6 & 11 & 29 \\
\hline $\begin{array}{l}\text { obsessive compulsive } \\
\text { disorder symptoms }\end{array}$ & 0.4 & 4 & 0.9 & 3 & 17 \\
\hline OCD & 0.2 & 3 & 0.7 & 2 & 33 \\
\hline
\end{tabular}

Our two most efficient keyword phrases included three elements, ocd, children, and symptoms, whereas our less efficient keyword phrases omitted one or more of these elements. In hindsight it seems obvious that a parent who was looking for information about children's OCD symptoms (all three elements) would be more likely to go to, and complete, a survey with those features than to one with only one or two of these features.

Our choice of conversion criterion, reaching the last page of the survey, was our unique criterion, so it is interesting to compare it to some of Google's measures of user experience, Quality Score (Q) and Click-Through Rate (CTR). A third user experience metric, suggested by some, is a composite, represented by the product of $Q$ and CTR, which is also listed in Table 2. How did our conversion-based measure compare to Google's user experience metrics? We correlated for the 10 keywords our Cnv1K measure with $Q$, CTR, and $Q^{*} C T R$ and obtained the following correlations, .32, .93, and .81, respectively. These results buttress the point that 
Professional Psychology: Research and Practice

(C) 2013 American Psychological Association DOI: 10.1037/a0034505

This article may not exactly replicate the final version published in the APA journal. It is not the copy of record.

keyword choice is very influential for Adwords outcome. In our experience, more detailed phrases worked better than general ones. For our specialized and single study, click-through rate was a surprisingly strong indicator of a keyword's success in leading to survey completion.

Google claims to reward good user experience by charging less for higher quality keywords. For our ten best keywords, the average cost per conversion is listed in rightmost column of Table 2. We found that better performing keywords were moderately associated with lower costs. The strongest association was between our cost of conversion, which correlated -.50 with the number of conversions we obtained per 1,000 impressions; low conversion rate accompanied higher costs. In a similar vein, higher costs were associated with lower quality scores, -.20 , and with lower click-through rates, -.27 . Thus, we did find that our best converting keywords had lower costs than did our poorly converting keywords.

\section{International Comparisons}

We wanted to know if we could successfully recruit from multiple countries, which is possible because Adwords enables the specification of targeted geographic locations. We chose 11 locations with significant English-speaking populations where our ads would display. We had no initial hypotheses about location differences in ad performance; however, we wanted to do a preliminary evaluation of location effects. The locations varied widely in their populations, so we needed a measure that would adjust for that variance. Our conversion measure per 1000 impressions would have been ideal, but with a total of 183 conversions, the sample sizes per location got quite small in some cases. As an alternative we have used CTR, which correlated highly with conversions/1K impressions, and in Figure 1 we present CTR by location. 
Professional Psychology: Research and Practice

(C) 2013 American Psychological Association DOI: 10.1037/a0034505

This article may not exactly replicate the final version published in the APA journal. It is not the copy of record.

Figure 1

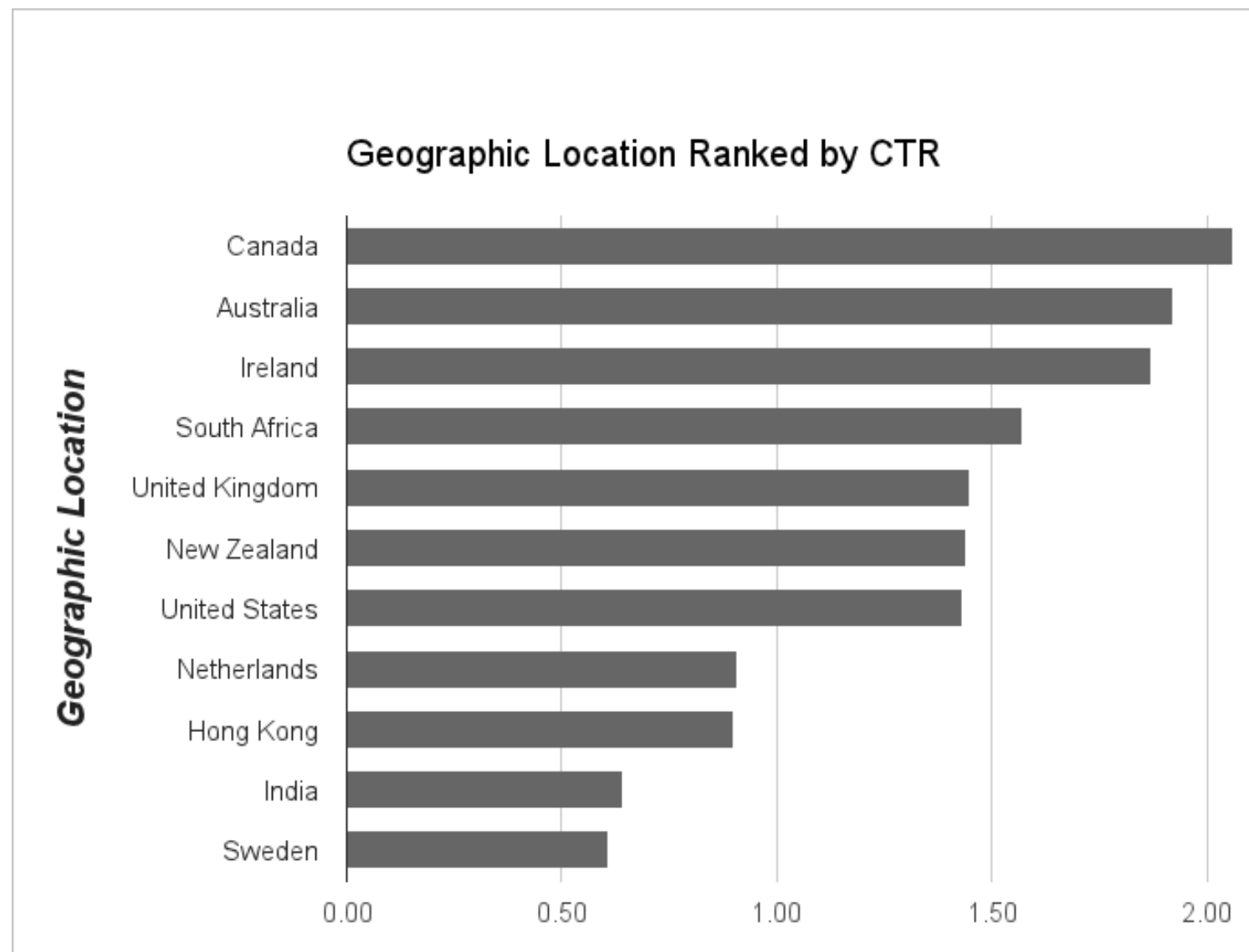

Click-Though Rate (\%)

We were able to recruit participants from all 11 locations, and Figure 1 shows considerable variability across locales ease in click-through rates. There are many possible factors that could influence that variation, such country-to-country differences in rates of OCD diagnosis. Regardless of which factors might be responsible, it seems likely that the cost of Adwords recruitment will vary widely across jurisdictions.

\section{Discussion}

Telepractice is inextricably tied to the internet because the web offers a very convenient vehicle for clients and practitioners to connect with each other from distant locations. In most cases, individuals will first seek help for a problem by searching online, so a professional who can easily be found by a web searcher has established an opening for a relationship with that seeker of help. Being found is the hard part. For example, an authoritative website that is rich with relevant content and that is linked to by many others will rank highly in a search. However, such web authority takes considerable time (months and years) and effort to develop. A quicker 
Professional Psychology: Research and Practice

(C) 2013 American Psychological Association DOI: 10.1037/a0034505

This article may not exactly replicate the final version published in the APA journal. It is not the copy of record.

alternative for being noticed online is to use pay-per click advertisements such as those implemented in Google Adwords campaigns.

We used an Adwords campaign to recruit the parents of children with OCD to our online research study in a proof-of-concept case study. We began with two questions: Could we successfully use Google adwords to be noticed by parents looking for information about childhood OCD? Further, assuming we were noticed, could we recruit an adequate sample for a reasonable cost? We think the answer to both questions is Yes.

Our use of Google Adwords to attract parents of OCD children yielded nearly 200 participants in 16 weeks for approximately $\$ 5000$. Under the conservative assumption that only 100 of the 200 participants will yield complete data, the cost of each analyzable data point would be roughly $\$ 50$. Further refinements in our ads and keywords would undoubtedly lower this amount.

Had we recruited locally in our city of 600,000 , it is highly unlikely that that we would have obtained such a large sample. More traditional advertising approaches, such as newspaper ads, would've been equally or more expensive, particularly if they were used repeatedly over time. Whether our online recruitment results constitute success is open to question, and we can not claim that similar results will extend to other projects and contexts. Nevertheless, we believe that the use of pay-per-click advertising offers a viable tool that could be useful for psychologists, both for research and practice. That said, we have learned that an Adwords strategy has a number of complexities that researchers and practitioners must consider, and it is to these complexities that we now turn.

When one starts an Adword campaign the cost can seem deceptively inexpensive. Really, only a dollar or two per click? That's cheap! However, we quickly learned that many clicks are needed to obtain the desired visitor action (a conversion), so the actual cost of a conversion was many times larger than the price for a click. Whether or not the total cost of a conversion is worth the expenditure depends on the relation between the cost of a conversion and the value of the user's action. A conversion can be defined by many different types of action. Our conversion was triggered by the visitor reaching the end of our survey, but one could define a conversion as the visitor submitting a Contact form. As long as some type of conversion is defined, the Adwords system computes the average number of advertising dollars spent to trigger it. Such conversion information can then be compared to some other standard, such as the amount of one's research grant or the added value of obtaining a new client. The larger point is that the PPC framework provides a great deal of detailed information about the cost and effectiveness of an advertising campaign.

Cost considerations are certainly important in determining which keyword terms and phrases one is going to use in a PPC campaign. Broad, general keywords attract interest from many advertisers, and such keywords are usually highly competitive and consequently more expensive. In contrast, narrowly defined, niche-related keyword phrases are typically less expensive and more effective. For example, as we saw in Table 2, keyword phrases like symptoms of ocd in children had much higher conversion rates that a more general keyword like $O C D$. In short, the cost of an adwords campaign will vary widely depending on how it is conducted. Campaigns built around a specific and focused set of keywords and phrases are 
Professional Psychology: Research and Practice

(C) 2013 American Psychological Association DOI: 10.1037/a0034505

This article may not exactly replicate the final version published in the APA journal. It is not the copy of record.

more likely to be cost effective in our view. At the other extreme, and adwords campaign for a broad psychological term like depression will be extremely expensive and likely less effective. Another cost, a hidden one, is the time that must be invested in monitoring and fine-tuning keywords and other campaign characteristics. Many experts recommend weekly reviews of adwords campaigns (Collins, 2012).

\section{Implications for Practitioners}

The preceding cost scenario means that Adwords campaigns are well suited for specialized niches, a fact that has important implications for the clinical psychologist. For example, it would undoubtedly be far more expensive for a psychologist to conduct a campaign around the keyword phrase anxiety treatment than for one based on the phrase fear of open spaces. Although there would be many fewer searchers for the latter phrase than for the former, a person looking for help on fear of open spaces who finds an ad specifically about that specific problem will be much more likely to click on that ad than on a less focused ad. A second benefit follows if the searcher arrives at a relevant website: Google assigns higher quality scores to keyword phrases with higher click-through-rates. Higher click-through rates and higher quality scores are, in turn, rewarded by Google with lower ad costs, so Adwords campaigns are well-suited to niche topics.

Because the costs of PPC advertising in a specific niche are generally lower than the costs of advertising in a general domain, a psychologist with a niche specialty could effectively use Adwords for being found by potential clients. This strength of pay-per-click online advertising dovetails nicely with the strengths of telepractice because specialized professionals can use telepractice tools to work with distant clients with rare problems. PPC helps connect client and professional; telepractice enables interaction between them. We suspect that the marriage of PPC and telepractice will contribute to more highly specialized practices, at least for some clinicians. A niche speciality that would have been impossible if based solely on local clients, could be more feasible when approached through telepractice. Adwords advertising could provide an important vehicle for client recruitment of non-local, telepractice clients. Of course, such online recruitment for specialized treatments should only be conducted by clinicians who are practicing within their established areas of expertise.

The preceding niche argument has a second side, one that is more relevant to a clinician who is not involved with telepractice. Although we chose to recruit from multiple countries, PPC advertising is not restricted to global searches. We could have had our ads displayed only to searchers located within a few miles of a specified location. This means that PPC campaigns can be focused on extremely small geographical areas, a feature that is particularly well-suited to practitioners who want to attract local clients. A local, geographically-limited campaign would mean that fewer searchers would see the ad, and there would be a corresponding decrease in the number of clicks (and cost). Such campaign could be practical for a professional with a local client catchment area.

The ability to restrict advertisements to specific locations could also be important for compliance with licensing laws. If one is only licensed to practice in one state, for example, it would be possible to restrict the appearance of ads to searchers in that state. Such location 
Professional Psychology: Research and Practice

(C) 2013 American Psychological Association DOI: 10.1037/a0034505

This article may not exactly replicate the final version published in the APA journal. It is not the copy of record.

constraints may be imperfect. For example a user's location at the time of search may not be the same as their legal residence. To guard against problems, the website content would need to provide clear information about licensing boundaries.

\section{Closing Comments}

Our experiences with adwords recruitment for a research survey are not necessarily predictive of results that may be obtained for other purposes. Indeed, we have found results to vary across our own research studies for non-clinical topics. We believe, nevertheless, that online PPC advertising may be applicable to be a relevant tool for many clinical researchers and practitioners. In Table 3 we provide an overview of the steps in setting up an adwords campaign. Supplementary information for each step can be readily found online, much of it in tutorials provided by Google.

\section{Table 3}

\section{Summary of Steps to Take in Using Google Adwords}

1. Articulate a problem for which you have information or a service.

2. Create a list of keywords and phrases that a lay person would use to search online for help with that problem.

3. Open an Adwords account and use Google's Keyword Planner to evaluate your keywords and to identify related terms.

4. Once you have identified your primary keywords, create a webpage that describes your services with relevant wording and concepts.

5. Create one or more text ads that speak to your searchers' problem.

6. Point each ad to the linked landing page (if you have services for different problems, create different ads and separate landing pages).

7. Identify an action you hope your user will take once they visit your landing page (e.g., initiate contact or download a pdf file).

8. Use the Adwords Conversion tool to create a conversion code, and embed it at your action button or location (e.g., on the Thank-you page of a survey).

9. For each of your keywords set the maximum amount you are willing to pay for a click.

10. Specify your daily and overall campaign budget.

11. Narrow the settings for the display of your ads to specific geographic locations or times of day (this decreases your competition and costs).

12. Switch on your campaign and try not to spend all your time watching for results.

13. Monitor results weekly, and adjust ads and keywords in response to the data, particularly data on conversions.

14. Discontinue poorly performing ads and keywords, and replace them with promising alternatives. Repeat steps 13 and 14.

The preceding sequence of actions is widely applicable, regardless of your purpose. However, we see some differences in the likely use of PPC advertising for research and 
Professional Psychology: Research and Practice

(C) 2013 American Psychological Association DOI: 10.1037/a0034505

This article may not exactly replicate the final version published in the APA journal. It is not the copy of record.

practice. In particular, PPC offers the researcher an opportunity to find farflung individuals who share a problem, perhaps one that is quite rare. Consequently, researcher PPC campaigns are likely to target geographically broad areas with specialized information or services. Most practicing psychologists, in contrast, treat a broad range of problems in clients from a relatively small local area. Stated differently, the researcher's niche is likely to be limited by the topic, and the clinician's niche is likely to limited by geography. PPC campaigns are flexible enough to accommodate both needs, either through niche-focused keywords for the researcher, or through location-focused impression displays for the practitioner.

A first encounter with the world of pay-per-click online advertising can produce a variety of reactions: amazement, dismay, and admiration. This entire system did not even exist 15 years ago, yet it has had a huge effect in many domains! One could spend a career mastering the subtleties and strategies involved with Adwords campaigns. Fortunately, as we have discovered from our proof-of-concept, it is feasible and practical to use Adwords to successfully recruit participants to clinically oriented research studies. We also believe that pay-per-click online advertising can also be used by practicing clinicians, in particular, to help local individuals find help from qualified professionals who are nearby.

\section{References}

Anderson, Chris (2006). The long tail: Why the future of business is selling less of more. New York: Hyperion.

Collins, D. (2012, May). Google AdWords: Stop losing and start exploiting (really). Presentation at Microconf 2012, Las Vegas. Retrieved May 29, 2013, from http://www.softwarepromotions.com/news/talking-google-adwords-2013-05-23/

Check and Understand (2013). Check and understand quality score. Retrieved May 22, 2013, from https://support.google.com/adwords/answer/2454010.

comScore (2013). comScore releases January 2013 U.S. search engine rankings. Retrieved May 17,2013 , from http://www.comscore.com/Insights/Press_Releases/2013/2/comScore_Releases_January 201 3 U.S. Search Engine Rankings.

Fox, S. \& Duggan, M. (2013). Pew Internet and American Life Project :Health Online 2013. Retrieved May 17, 2013, from http://www.pewinternet.org/Reports/2013/Health-online/Summary-of-Findings.aspx.

Google, Inc. (2013). 2013 financial tables. Retrieved May 17, 2013, from http://investor.google.com/financial/tables.html. 
Professional Psychology: Research and Practice

(C) 2013 American Psychological Association DOI: 10.1037/a0034505

This article may not exactly replicate the final version published in the APA journal. It is not the copy of record.

Hitstudios (2013). Google Adwords highest paid keywords. Retrieved May 19, 2013, from http://hitstudios.net/marketing-tips/google-adwords-top-paying-keyword-list-2012-2013.

Jones, R. B.; Goldsmith, L, Williams, C. J., Boulos, M. N. K. (2012). Accuracy of geographically targeted internet advertisements on Google AdWords for recruitment in a randomized trial. Journal of medical internet research, 14, 1439-4456. 